\title{
Is segmentectomy indicative for small-sized non-small cell lung cancer in the basal segments with a small ground-glass opacity component?
}

\author{
Terumoto Koike, Akihiro Nakamura, Yuki Shimizu, Tatsuya Goto, Akihiko Kitahara, Seijiro Sato, \\ Masanori Tsuchida \\ Division of Thoracic and Cardiovascular Surgery, Niigata University Graduate School of Medical and Dental Sciences, Niigata, Japan \\ Correspondence to: Terumoto Koike, MD, PhD. Division of Thoracic and Cardiovascular Surgery, Niigata University Graduate School of Medical and \\ Dental Sciences, 1-757 Asahimachi-dori, Chuo-ku, Niigata 951-8510, Japan. Email: t-koike@kj8.so-net.ne.jp. \\ Provenance: This is an invited Editorial commissioned by Section Editor Dr. Akira Hamada (Department of Cardiovascular, Thoracic and Pediatric \\ surgery, Faculty of Medicine, Yamagata University, Yamagata, Japan). \\ Comment on: Nishio W, Yoshimura M, Maniwa Y, et al. Re-Assessment of Intentional Extended Segmentectomy for Clinical T1aN0 Non-Small Cell \\ Lung Cancer. Ann Thorac Surg 2016;102:1702-10.
}

Submitted Aug 16, 2017. Accepted for publication Aug 31, 2017.

doi: $10.21037 /$ jtd.2017.09.101

View this article at: http://dx.doi.org/10.21037/jtd.2017.09.101

The current issue of The Annals of Thoracic Surgery has published the remarkable work of Nishio and associates (1) which contributes to a deeper understanding of the locoregional control and postoperative long-term outcomes after an intentional extended segmentectomy for peripheral non-small cell lung cancer (NSCLC) of $\leq 2 \mathrm{~cm}$. The report compares segmentectomy to lobectomy, which is the standard surgical procedure, with a special focus on the effects of radiological characteristics and segment locations on locoregional control and postoperative survival in patients with those NSCLC.

The authors have retrospectively reviewed 164 patients who underwent intentional extended segmentectomy and 73 patients who underwent lobectomy (1). All the patients had cT1aN0M0 disease according to the 7th edition of the TNM classification (2). Based on the thin-section computed tomography (CT) findings, patients in the segmentectomy and lobectomy groups were categorized as $\leq 0.5$ or $>0.5$ of a consolidation to maximum tumor diameter ratio (C/T). In 47 patients with $\leq 0.5$ of $\mathrm{C} / \mathrm{T}$ (46 and 1 patients in the segmentectomy and lobectomy groups, respectively), pathological examination did not find a nonadenocarcinoma or lymph node metastasis. During the median followup period of 109 months, no recurrence was observed. In 190 patients with $>0.5$ of C/T, 59 patients from the segmentectomy and lobectomy groups were matched using the propensity-matched model. Between the propensitymatched segmentectomy and lobectomy groups, no significant differences were observed both in overall survival (OS) and locoregional recurrence-free survival $(\mathrm{P}=0.234$ and $\mathrm{P}=0.082$, respectively). A multivariate analysis of all the 190 patients with $>0.5$ of $\mathrm{C} / \mathrm{T}$ revealed the surgical procedure to be a significant risk factor for locoregional recurrence-free probability $(\mathrm{P}=0.020)$, while only age and $\mathrm{C} / \mathrm{T}$ were identified as independent prognostic factors for locoregional recurrence-free survival $(\mathrm{P}=0.019$ and $\mathrm{P}=0.012$, respectively). In 118 patients with $>0.5$ of $\mathrm{C} / \mathrm{T}$ who underwent segmentectomy, locoregional recurrence was relatively high in patients with a tumor in the basal segment [ 7 patients in the right upper lobe $(n=32), 6$ in the left upper lobe ( $\mathrm{n}=38), 1$ in the bilateral superior segment $(n=24)$, and 9 in the bilateral basal segments $(n=24)]$. In the 70 patients with a tumor in the bilateral lower lobe, the 5- and 10-year locoregional recurrence-free survival rates were $91.7 \%$ and $69.5 \%$, respectively, for the lower lobe superior segmentectomies, $62.5 \%$ and $40.7 \%$, respectively, for segmentectomies of the basal segments, and $100.0 \%$ and $76.4 \%$, respectively, for lobectomies $(\mathrm{P}=0.011)$. No significant difference in locoregional recurrence-free survival was observed between segmentectomies and lobectomies of the right or left upper lobe $(\mathrm{n}=67 ; \mathrm{P}=0.075$ and $\mathrm{n}=53 ; \mathrm{P}=0.438$, respectively). 
The Lung Cancer Study Group performed a phase III prospective randomized study of limited resection versus lobectomy for small-sized NSCLC and demonstrated that limited resection had a threefold higher locoregional recurrence rate (3). Based on these findings lobectomy has been the standard surgical procedure for NSCLC patients regardless of the tumor size. However, because of developments in the imaging technology and CT screening, increasing number of smaller and more indolent NSCLCs are detected (4). Previously, several prospective trials of intentional limited resection for patients with NSCLC of $\leq 2 \mathrm{~cm}$ have been conducted $(5,6)$. Most patients in these studies underwent segmentectomy. These patients demonstrated local control and postoperative survival for intentional limited resection were comparable to those seen in patients undergoing lobectomy. However, in these series, since the studies were non-randomized trials and the NSCLC patients for sublobar resection were selected based solely on radiological small tumor size, the appropriate and inappropriate subgroups for intentional limited resection were not known.

Numerous studies have documented strong correlations between pure or part solid ground-glass nodule (GGN) on CT, and adenocarcinoma in situ or minimally invasive adenocarcinoma $(7,8)$. Pathological lung adenocarcinoma in situ presents with ground-glass opacity (GGO) on CT due to the preservation of existing lung structures such as the alveoli, bronchi, and vessels $(9,10)$. A tumor with a high proportion of GGO component or low C/ $\mathrm{T}$ is therefore, highly indicative a high proportion of noninvasive adenocarcinoma component. Suzuki et al. reviewed 545 patients with resected lung adenocarcinoma of $\leq 3 \mathrm{~cm}$ on a preoperative CT and demonstrated a strong correlation between a tumor $\leq 2 \mathrm{~cm}$ with $\mathrm{C} / \mathrm{T} \leq 0.25$ and pathological noninvasive lung cancer, which was defined as a lung adenocarcinoma without nodal involvement, vascular invasion, or lymphatic invasion (11). Patients with lung adenocarcinoma of $\leq 2 \mathrm{~cm}$ and a $\leq 0.25$ of $\mathrm{C} / \mathrm{T}$ demonstrated excellent overall and relapse-free survivals after surgical treatment (the 5-year overall and relapse-free survivals of $97.1 \%$ and $97.1 \%$, respectively) (12). Therefore, small-sized peripheral NSCLC with low C/T was considered an appropriate subgroup for limited resection. Many studies of intentional limited resection using an intraoperative frozen-section examination addressed these tumors $(13,14)$. Even though the majority of patients underwent wedge resection, excellent surgical outcomes with no evidence of recurrence were demonstrated.
Therefore, it seems reasonable that in the study by Nishio et al., the 47 patients (46 and 1 patients in the segmentectomy and lobectomy groups, respectively) with a tumor $\leq 2 \mathrm{~cm}$ with $\mathrm{C} / \mathrm{T} \leq 0.5$ showed excellent postoperative outcomes without any recurrences (1). Pathological examination of these 47 patients revealed that none of them had lymph node metastasis, and a few had a pleural, vascular, or lymphatic invasion.

In the study by Nishio et al., among 190 patients (118 and 72 patients in the segmentectomy and lobectomy groups, respectively) who had tumors with $\mathrm{C} / \mathrm{T}>0.5,32$ patients in the segmentectomy group and 11 in the lobectomy group developed recurrence during the median follow-up periods of 107 and 84 months, respectively (1). The pathological examination showed that 46 (24\%), 42 (22\%), and $41(22 \%)$ patients had a pleural, vascular, and lymphatic invasion, respectively. Thus, tumors with $\mathrm{C} / \mathrm{T}>0.5$ were thought to be more aggressive compared to those with $\mathrm{C} / \mathrm{T} \leq 0.5$. The 5 - and 10 -year OS rates for the propensitymatched segmentectomy group $(\mathrm{C} / \mathrm{T}>0.5)$ were $91.5 \%$ and $74.9 \%$, respectively, versus $94.8 \%$ and $79.3 \%$, respectively, for the propensity-matched lobectomy group $(\mathrm{P}=0.234)$. Previously, we conducted a retrospective comparative study of segmentectomy versus lobectomy in NSCLC patients with radiologically pure solid tumors $(1.0$ of $\mathrm{C} / \mathrm{T})$ of $\leq 2 \mathrm{~cm}$ (15). In the propensity score matched segmentectomy and lobectomy groups (87 patients per group), the 5- and 10 year OS rates were $84 \%$ versus $85 \%$ and $63 \%$ versus $66 \%$, respectively $(\mathrm{P}=0.767)$. Even though tumors with 1.0 of $\mathrm{C} / \mathrm{T}$ should have been more aggressive disease than those with $\mathrm{C} / \mathrm{T}>0.5$, the results of both the studies were comparable, suggesting that both segmentectomy and lobectomy had similar postoperative outcomes in these study cohorts. However, Nishio et al. have suggested the possibility of an inferior locoregional control after segmentectomy compared to lobectomy in their study (1). The locoregional recurrence-free survival rates in the propensity-matched segmentectomy group were relatively lower compared to the propensity-matched lobectomy group. The 5- and 10-year locoregional-free survival rates were $76.3 \%$ versus $91.5 \%$ and $67.2 \%$ versus $77.1 \%$, respectively, in the segmentectomy and lobectomy groups, respectively $(\mathrm{P}=0.082)$. The multivariate analysis identified surgical procedure (segmentectomy or lobectomy) as a significant independent prognostic factor for locoregionalfree probability (not for locoregional-free survival). These findings by Nishio et al. suggested that in patients with small-sized NSCLC of high C/T on preoperative CT, the 
long-term outcome following segmentectomy might be comparable to that following lobectomy, despite a higher risk of locoregional recurrence. In our previous study comparing segmentectomy and lobectomy in patients with small-sized pure solid NSCLC (15), although the results of locoregional recurrence-free survival and probability were not presented, the relatively higher incidence of locoregional recurrence after segmentectomy was indicated when compared to lobectomy. During the follow-up periods, while 9 patients were identified with locoregional recurrence as a first recurrence site in the propensitymatched segmentectomy group $(\mathrm{n}=87), 5$ patients were identified in the propensity-matched lobectomy group $(\mathrm{n}=87)$. On the other hand, while 14 patients were identified with distant recurrence (three patients had both locoregional and distant) as a first recurrence site in the propensitymatched segmentectomy group, whereas 12 patients were identified in the propensity-matched lobectomy group. One possible explanation for the non-inferior outcomes after segmentectomy despite the higher incidence of locoregional recurrence in the previous studies could be that regardless of the extent of pulmonary resection, the postoperative recurrences at distant sites affect postoperative survival more than those at locoregional sites. Some patients might simultaneously develop locoregional and distant recurrences as the first recurrence site, and in some patients who develop locoregional recurrences, surgery and radiation therapy might be effective and could provide survival benefits. Previously, we presented some cases of repeated lung resection of ipsilateral lung cancer that was detected after segmentectomy for primary lung cancer, and this study included two cases of repeated lung resection for locoregional recurrence after segmentectomy (16). The one of the two patients underwent right superior segmentectomy for the recurrence at the surgical margin 58 months after right basal segmentectomy for primary lung adenocarcinoma. The patient did not undergo any adjuvant chemotherapy and has been alive for 111 months after the first segmentectomy with no evidence of recurrence. We need to recognize that some discrepancies may exist between postoperative locoregional control and survival in NSCLC patients after limited resection. In fact, even in the Lung Cancer Study Group study, no significant difference was observed in the postoperative survival among patients who underwent limited resection and lobectomy despite an approximately threefold higher locoregional recurrence rate associated with limited resection (3).

Nishio et al. have reported a significantly high frequency of locoregional recurrences in selective basal segmentectomies, suggesting that differences in locoregional recurrence-free survival could depend on the segment locations (1). While some studies have reported no difference in locoregional recurrence and/or survival based upon lobar location in patients with clinical stage IA NSCLC who underwent limited resection and pathologic stage I/II NSCLC who underwent pulmonary resection $(17,18)$, there are others reporting that tumors resected by segmentectomy from the right upper lobe or left upper division segment were more likely to recur locally compared to those from the remaining segments (19). In our previous study of the risk factor analysis of locoregional recurrence after sublobar resection in patients with clinical stage IA NSCLC (18), there was no significant difference in locoregional recurrence-free probability between the tumor located lobes but the 5 -year locoregional recurrence-free probabilities were relatively lower in tumors located in the lower lobes $(76.6 \%, 81.7 \%, 86.2 \%$, and $90.1 \%$, for the left lower lobe, the right lower lobe, the right upper/middle lobe, and the left upper lobe, respectively). Although the association between the segment locations and postoperative locoregional recurrence and/or survival is still unknown, surgeons should consider the anatomical difficulty of ensuring resection margin and of full hilar lymph node dissection in some of the segmentectomies as Nishio et al. described in their report (1).

Nishio et al. have reported an association between the $\mathrm{C} / \mathrm{T}$ on preoperative $\mathrm{CT}$ or the segment locations and postoperative locoregional control and/or outcomes in patients with NSCLC of $\leq 2 \mathrm{~cm}$ who underwent intentional extended segmentectomy. Many previous studies have suggested risk factors for postoperative locoregional recurrence and/or poor outcomes after limited resection, such as male sex, severe smoking history, high $\mathrm{C} / \mathrm{T}$ on preoperative $\mathrm{CT}$, high maximum standardized uptake values on F-18-fluorodeoxyglucose positron emission tomography/ CT, tumor histology other than adenocarcinoma, visceral pleural invasion, and lymphatic permeation (15,18,20-23). Limited resection for small-sized NSCLC patients with these risk factors is still controversial. We believe that in future, ongoing prospective randomized trials of limited resection versus lobectomy in patients with small-sized peripheral NSCLC, such as the CALGB 140503 (24) and the Japan Clinical Oncology Group 0802/West Japan Oncology Group 4607L (25) will answer these questions. However, we still need time to obtain, assess, and interpret the results of these trials. Although we recently reported the results of a randomized controlled multicenter study of 
limited resection versus lobectomy for peripheral NSCLC of $\leq 2 \mathrm{~cm}$ (23), the sample size was too small for subset analysis. Therefore, there is a need to agree upon an indication of intentional limited resection for small-sized NSCLC patients with risk factors for locoregional recurrence and/ or poor survival. Retrospective studies of limited resection versus lobectomy, such as the study by Nishio et al. (1) may be helpful in providing a better understanding and decisions for intentional limited resection in patients with small-sized NSCLC.

\section{Acknowledgements}

None.

\section{Footnote}

Conflicts of Interest: The authors have no conflicts of interest to declare.

\section{References}

1. Nishio W, Yoshimura M, Maniwa Y, et al. Re-Assessment of Intentional Extended Segmentectomy for Clinical T1aN0 Non-Small Cell Lung Cancer. Ann Thorac Surg 2016;102:1702-10.

2. Sobin LH, Gospodarowicz MK, Wittekind C. UICC International union against cancer. TNM Classification of Malignant Tumours. 7th Edition 2009.

3. Ginsberg RJ, Rubinstein LV. Randomized trial of lobectomy versus limited resection for T1 N0 non-small cell lung cancer. Lung Cancer Study Group. Ann Thorac Surg 1995;60:615-22; discussion 622-3.

4. Donington JS. Point: Are limited resections appropriate in non-small cell lung cancer? Yes. Chest 2012;141:588-90.

5. Koike T, Yamato Y, Yoshiya K, et al. Intentional limited pulmonary resection for peripheral T1 N0 M0 small-sized lung cancer. J Thorac Cardiovasc Surg 2003;125:924-8.

6. Okada M, Koike T, Higashiyama M, et al. Radical sublobar resection for small-sized non-small cell lung cancer: a multicenter study. J Thorac Cardiovasc Surg 2006;132:769-75.

7. Austin JH, Garg K, Aberle D, et al. Radiologic implications of the 2011 classification of adenocarcinoma of the lung. Radiology 2013;266:62-71.

8. Lee KH, Goo JM, Park SJ, et al. Correlation between the size of the solid component on thin-section CT and the invasive component on pathology in small lung adenocarcinomas manifesting as ground-glass nodules. J Thorac Oncol 2014;9:74-82.

9. Aoki T, Nakata H, Watanabe H, et al. Evolution of peripheral lung adenocarcinomas: CT findings correlated with histology and tumor doubling time. AJR Am J Roentgenol 2000;174:763-8.

10. Asamura H, Suzuki K, Watanabe S, et al. A clinicopathological study of resected subcentimeter lung cancers: a favorable prognosis for ground glass opacity lesions. Ann Thorac Surg 2003;76:1016-22.

11. Suzuki K, Koike T, Asakawa T, et al. A prospective radiological study of thin-section computed tomography to predict pathological noninvasiveness in peripheral clinical IA lung cancer (Japan Clinical Oncology Group 0201). J Thorac Oncol 2011;6:751-6.

12. Asamura H, Hishida T, Suzuki K, et al. Radiographically determined noninvasive adenocarcinoma of the lung: survival outcomes of Japan Clinical Oncology Group 0201. J Thorac Cardiovasc Surg 2013;146:24-30.

13. Yoshida J, Nagai K, Yokose T, et al. Limited resection trial for pulmonary ground-glass opacity nodules: fifty-case experience. J Thorac Cardiovasc Surg 2005;129:991-6.

14. Koike T, Togashi K, Shirato T, et al. Limited resection for noninvasive bronchioloalveolar carcinoma diagnosed by intraoperative pathologic examination. Ann Thorac Surg 2009;88:1106-11.

15. Koike T, Kitahara A, Sato S, et al. Lobectomy Versus Segmentectomy in Radiologically Pure Solid SmallSized Non-Small Cell Lung Cancer. Ann Thorac Surg 2016;101:1354-60.

16. Tsuchida M, Goto T, Kitahara A, et al. Repeated lung resection of ipsilateral lung cancer that is detected after segmentectomy for primary lung cancer. J Thorac Oncol 2017;12:S1399.

17. Puri V, Garg N, Engelhardt EE, et al. Tumor location is not an independent prognostic factor in early stage nonsmall cell lung cancer. Ann Thorac Surg 2010;89:1053-9.

18. Koike T, Koike T, Yoshiya K, et al. Risk factor analysis of locoregional recurrence after sublobar resection in patients with clinical stage IA non-small cell lung cancer. J Thorac Cardiovasc Surg 2013;146:372-8.

19. Sienel W, Stremmel C, Kirschbaum A, et al. Frequency of local recurrence following segmentectomy of stage IA nonsmall cell lung cancer is influenced by segment localisation and width of resection margins--implications for patient selection for segmentectomy. Eur J Cardiothorac Surg 2007;31:522-7.

20. Koike T, Koike T, Yamato Y, et al. Prognostic predictors in 
non-small cell lung cancer patients undergoing intentional segmentectomy. Ann Thorac Surg 2012;93:1788-94.

21. Tsutani Y, Miyata Y, Nakayama H, et al. Oncologic outcomes of segmentectomy compared with lobectomy for clinical stage IA lung adenocarcinoma: propensity score-matched analysis in a multicenter study. J Thorac Cardiovasc Surg 2013;146:358-64.

22. Okada M, Mimae T, Tsutani Y, et al. Segmentectomy versus lobectomy for clinical stage IA lung adenocarcinoma. Ann Cardiothorac Surg 2014;3:153-9.

23. Koike T, Koike T, Sato S, et al. Lobectomy and limited resection in small-sized peripheral non-small cell lung

Cite this article as: Koike T, Nakamura A, Shimizu Y, Goto T, Kitahara A, Sato S, Tsuchida M. Is segmentectomy indicative for small-sized non-small cell lung cancer in the basal segments with a small ground-glass opacity component? J Thorac Dis 2017;9(10):3501-3505. doi:10.21037/jtd.2017.09.101 cancer. J Thorac Dis 2016;8:3265-74.

24. National institutes of health. National cancer institute. Calgb-140503. Phase iii randomized study of lobectomy versus sublobar resection in patients with small peripheral stage ia non-small cell lung cancer. Available online: http:// www.Cancer.Gov/clinicaltrials/calgb-140503. Accessed: Mar 16, 2010.

25. Nakamura K, Saji H, Nakajima R, et al. A phase III randomized trial of lobectomy versus limited resection for small-sized peripheral non-small cell lung cancer (JCOG0802/WJOG4607L). Jpn J Clin Oncol 2010;40:271-4. 